\title{
The Hubble Constant
}

\author{
Jeremy Mould \\ Research School of Astronomy \& Astrophysics, Australian National University, \\ Mount Stromlo Observatory, Weston, ACT 2611, Australia \\ jrm@mso.anu.edu.au \\ Received 1999 July 29, accepted 1999 October 17
}

\begin{abstract}
With the completion of the Hubble Space Telescope (HST) Key Project on the Extragalactic Distance Scale, it is interesting to form the dimensionless quantity $H_{0} t_{0}$ by multiplying the Hubble Constant by the age of the Universe. In a matter dominated decelerating Universe with a density exceeding $0 \cdot 26$ of the critical value, $H_{0} t_{0}<1$; in an accelerating Universe with the same $\Omega_{m}=0 \cdot 26$, but dominated by vacuum energy with $\Omega_{V} \geq 1-\Omega_{m}, H_{0} t_{0} \geq 1$. If the first globular clusters formed $10^{9}$ years after the Big Bang, then with $95 \%$ confidence $H_{0} t_{0}=1 \cdot 0 \pm 0 \cdot 3$. The classical Einstein-de Sitter cosmological model has $H_{0} t_{0}=\frac{2}{3}$.
\end{abstract}

Keywords: cosmology: distance scale-galaxies: formation

\section{Introduction}

In a uniformly expanding Universe in which the solution to the field equations of General Relativity is consistent with the large scale distribution of matter, the dimensionless time derivative of the scale factor is $H$ and its current value $H_{0}$ is the Hubble Constant. The HST Key Project on the Extragalactic Distance Scale was announced in 1984 and called for proposals to measure the Hubble Constant to an accuracy of $10 \%$. This project was recently brought to a successful conclusion (Mould et al. 1999).

Following the analysis of Hipparcos parallaxes of nearby subdwarf stars, the uncertainty in the age of the Universe, $t_{0}$, is of a similar order. If the second derivative of the scale factor were zero, the expansion of the Universe would be steady and $H_{0}=t_{0}^{-1}$. This paper examines the observational constraints on $H_{0} t_{0}$ and the implications for the global dynamics of the Universe.

\section{Results of the Key Project}

The history of the measurement of the Hubble Constant is described by Huchra (1999). Kennicutt, Freedman \& Mould (1995) outlined the measurement of Cepheid distances for a set of galaxies within 25 $\mathrm{Mpc}$ and the subsequent calibration of secondary distance indicators to reach beyond $100 \mathrm{Mpc}$, where the thermal velocities of galaxies are much less than their recession velocities viewed from our location. Calibration of the Tully-Fisher relation was reported by Sakai et al. (1999). Calibration of the similar dynamical relation for elliptical galaxies (the Fundamental Plane) is described by Kelson et al. (1999). Calibration of the resolvability of earlytype galaxies through their fluctuation in surface brightness has been published by Ferrarese et al. (1999) and Tonry et al. (1999). Calibration of the supernova standard candle has been accomplished by Gibson et al. (1999) and Tammann (1998 and references therein). These constraints on the Hubble Constant have been combined by Mould et al. (1999), who find

$$
\begin{aligned}
H_{0} & =68 \pm 6 \mathrm{~km} / \mathrm{s} / \mathrm{Mpc} \\
& =\left[(14 \cdot 3 \pm 1 \cdot 4) \times 10^{9} \mathrm{yr}\right]^{-1}
\end{aligned}
$$

The quoted uncertainty is a $1 \sigma$ error.

\section{Age of the Universe}

The age of the first globular clusters to form in the Milky Way has been reviewed by Mould (1998). The error budget, like that for the Hubble Constant, is a list of daunting length. The distances of the clusters, their chemical composition, the photometry of their main sequence turnoff stars, their reddening, and the effect of undetected binary stars all contribute to the observational uncertainty in determining where the stars leave the main sequence. Stellar structure models transform this measurement into an estimate of how long such stars take to reach this evolutionary state. The treatment of convective energy transport and the sedimentation of helium in such old stars are unconstrained by observations and must be included in the uncertainties too.

The most recent results are by Carretta et al. (1999):

$$
\begin{aligned}
t_{0}= & (13 \cdot 2 \pm 2 \cdot 9) \times 10^{9} \mathrm{yr} \\
& 95 \% \text { confidence limits } .
\end{aligned}
$$



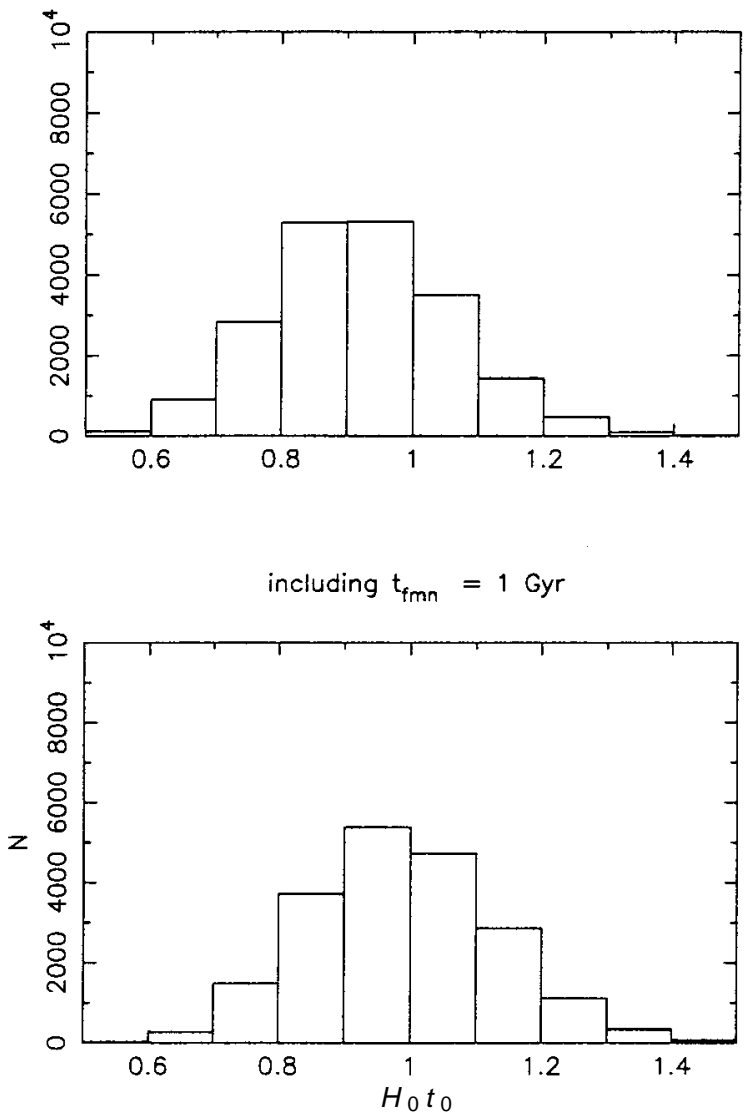

Figure 1-Probability distribution of the product of $H_{0}$ and $t_{0}$ with their associated uncertainties. Above: with $t_{f}=0$. Below: with $t_{f}=1$ Gyr.

This is consistent with all other work stemming from post-Hipparcos definition of the subdwarf main sequence and its fitting to the main sequences of globular clusters to measure their distances (Reid 1997; Chaboyer et al. 1998; Gratton et al. 1998; Pont et al. 1998). Carretta et al. take the extra step of checking that the RR Lyrae distance of the Large Magellanic Cloud, which follows from this, is consistent with the LMC distance assumed in the Key Project. In all this work the first globular clusters are taken to be those formed in the chemically most pristine epoch of the Galaxy.

Globular cluster ages are a lower bound on the age of the Universe. How close a bound is this? How early did globular cluster formation begin? The earliest conceivable time is $10^{6} \mathrm{yr}$ after the Big Bang, when the Jeans mass had fallen to $10^{6} \mathrm{M}_{\odot}$. By $10^{7}$ yr the intergalactic medium had reached a temperate $300 \mathrm{~K}$. Quite possibly the Universe waited $10^{8} \mathrm{yr}$, which is a dynamical time for galaxies. The first observational constraint one can cite is the work of Pahre (1997) who found $z_{f}>5$ for the major star formation epoch in elliptical galaxies in his study of the evolution of the Fundamental Plane. This corresponds to a time of formation $t_{f}$ $<1 \times 10^{9}$ yr in typical cosmologies.

\section{Constraints on Decelerating Cosmologies}

In a steadily decelerating Universe, which is matter dominated but open with a density ratio to the critical density $\Omega>0 \cdot 26$, we have $H_{0} t_{0}<1$ (Peacock 1999). A Universe which is accelerating now, and has $\Omega>0 \cdot 26$ and a vacuum energy density ratio $\Omega_{V} \geq 0 \cdot 74$, has $H_{0} t_{0} \geq 1$ (Carroll, Press \& Turner 1992). The uncertainty in the product of equations (1) and (2) can be calculated from the normally distributed errors in $H_{0}$ and $t_{0}$. Figure 1 shows the probability distribution for $H_{0} t_{0}$ for two assumptions: $t_{f}=0$ and 1 Gyr.

\section{Sharpening the Constraint}

Significant reductions in the error budget for the Key Project can be achieved through infrared measurements of the Cepheids. This work is in progress with NICMOS (Freedman et al. 2000) and stands to improve our knowledge of the chemical composition sensitivity of the Cepheid period luminosity relation and the extinction correction of the Cepheid distances. Extension of the range of the Cerro Calan/CTIO supernova survey (Hamuy et al. 1996) and the Mount Stromlo Abell cluster supernova survey (Reiss et al. 1998) will ensure that $H_{0}$ measured locally is as close as needs be to the global value. Improved photometric calibration will reduce a most unwelcome contribution to the Key Project's error budget (Mould et al. 1999).

In the longer term constraints on $H_{0}$ will emerge from the study of the surface of last scattering of the cosmic microwave background (CMB). The CBI and MAP missions expect to achieve results comparable in precision to the Key Project, but PLANCK plans to reach $\delta H / H<1 \%$. More conventionally, SIM will measure such accurate Cepheid parallaxes that the Key Project's dependence on the LMC for the basic reference distance will be lifted. Additional supernova calibrators can be expected to halve the uncertainty in the Hubble Constant over the lifetime of the HST. And SIM's accurate globular cluster parallaxes will halve the uncertainty in their ages.

Although future work on the CMB promises to constrain all the cosmological parameters to great accuracy, the surface of last scattering features at least as much interesting physics as the interiors of globular cluster stars. It will therefore be useful to pursue the conventional constraints on $H_{0} t_{0}$ as a parallel consistency check.

\section{Acknowledgments}

I wish to acknowledge my fellow team members in the $H_{0}$ Key Project. Team members include Shaun M. Hughes, Fabio Bresolin, Shoko Sakai, Barry F. Madore, Robert Hill, Laura Ferrarese, Holland C. Ford, Garth D. Illingworth, Daniel Kelson, Wendy Freedman, Robert Kennicutt, John A. Graham, 
Nancy Silbermann, Lucas Macri, Randy Phelps, Brad Gibson, John G. Hoessel, Mingsheng Han, John P. Huchra, Anne Turner, Abhijit Saha, Kim Sebo, and Peter B. Stetson. Much of the work presented in this paper is based on observations with the NASA/ESA Hubble Space Telescope, obtained by the Space Telescope Science Institute which is operated by AURA Inc under NASA contract No. 5-26555.

\section{References}

Carretta, E., Gratton, R., Clementini, G., \& Fusi Pecci, F. 1999, astro-ph 9902086

Carroll, S., Press, W., \& Turner, E. 1992, ARA\&A, 30, 409

Chaboyer, B., Demarque, P., Kernan, P. J., \& Krauss, L. M. 1998, ApJ, 494, 96

Ferrarese, L., et al. 1999, ApJ 529, 745

Freedman et al. 2000, in preparation

Gibson, B., et al. 1999, ApJ, 529, 723
Gratton, R., et al. 1998, ApJ, 491, 749

Hamuy, M., Phillips, M., Suntzeff, N., Schommer, R., Maza, J., \& Aviles, R. 1996, AJ, 112, 2398

Huchra, J. 1999, http://cfawww.harvard.edu/ huchra

Kelson, D., et al. 1999, ApJ, 529, 768

Kennicutt, Jr, R. C., Freedman, W. L., \& Mould, J. R. 1995, AJ, 110, 1476

Mould, J. 1998, Nature, 395, A20

Mould, J., et al. 1999, ApJ, 529, 786

Pahre, M. 1997, PhD thesis, Caltech

Peacock, J. 1999, Cosmological Physics (Cambridge Univ. Press).

Pont, M., Mayor, M., Turon, C., \& VandenBerg, D. 1998, A\&A, 329, 87

Reid, I. N. 1997, AJ, 114, 161

Reiss, A., Germany, L., Schmidt, B., \& Stubbs, C. 1998, AJ, 115,26

Sakai, S., et al. 1999, ApJ, 529, 698

Tammann, G. 1998, Proc. 8th Marcel Grossman Symposium, ed. T. Piran (Singapore: World Scientific), in press

Tonry, J., et al. 1999, ApJ, 530, 625 